\title{
Evaluation of Percutaneous Tendoachilles Tenotomy as an Outpatient Procedure in Clubfoot Treatment by Ponseti Method
}

Adel Abdelazim Ahmad Salem ${ }^{1}$, Khaled Edris Abdelrahman', Fahmi Subhi Alghazawi ${ }^{* 2}$, Mahmoud Elsayed Elbadawy Thabet ${ }^{1}$

${ }^{1}$ Orthopedic Department, Faculty of medicine, Zagazeg University, Sharkia, Egypt.

${ }^{2}$ Orthopedic Department, Faculty of medicine, Gharyan University, Gharyan, Libya.

*Corresponding Author: Fahmi Subhi Alghazawi, Mobile: 00218944629813, E-mail: algezawy2018@gmail.com

\begin{abstract}
Background: Clubfoot is a relatively common congenital foot deformity. Ponseti demonstrated correction of clubfoot in infants using manipulation followed by application of well-molded, long-leg plaster casts. Objective: In the current study, we evaluated the efficacy and safety of percutaneous Achilles tenotomy as an office procedure during the Ponseti technique in the correction of CTEV deformity of the foot. Patients and Methods: This randomized clinical trial study was conducted in the Orthopedic Outpatient Clinic of Zagazig University Hospitals on 12 patients with idiopathic clubfoot managed with the Ponseti technique during the period from April 2020 to September 2020. Results: This study showed, 13 feet had a favorable outcome (8 cases $(53.3 \%)$ excellent and 5 cases good 33.3\%) and 2 feet had fair outcome (13.3); these 2 feet had a relapse (13.3\%), one foot relapsed equinus deformity and one adducts foot deformity, The equinus deformity patient responded to repeated tendo-Achilles tenotomy and further Ponseti casting and adductus foot deformity responded to adductor tenotomy and cast. Both two cases obtained satisfactory outcomes after the completion of treatment. Conclusion: Achilles tenotomy as an out-patient procedure using topical and/or local anesthesia is a safe procedure and is an important step in the successful treatment of congenital clubfoot. Keywords: Clubfoot, Ponseti method, Achilles tenotomy.
\end{abstract}

\section{INTRODUCTION}

Clubfoot, also termed congenital talipes equinovarus (CTEV), is a common foot abnormality, in which the foot points downward (equinus) and forefoot inward (metatarsus adductus) hindfoot in (varus) midfoot in (cavus). The condition is present at birth. It is occurring in about one in every 1000 live births $^{(\mathbf{1})}$.

Clubfoot deformity may be associated with myelodysplasia, arthrogryposis, or multiple congenital abnormalities, but is most commonly an isolated birth defect and considered idiopathic ${ }^{(2)}$. The prevalence of additional congenital anomalies or chromosomal abnormalities in patients with clubfoot varies substantially across studies, depending on the population, and ranges from $24 \%$ to $50 \%{ }^{(3)}$.

Clubfoot is an embryonic malformation. A normally developing foot turns into a clubfoot during the second trimester of pregnancy. As regards the pathology, according to Turco, the talus is forced into the equinus by the underlying calcaneus and navicular, whereas the head and neck of the talus have deviated medially. The calcaneus is inverted under the talus, with the posterior end displaced upward and laterally, and the anterior end displaced downward and medially ${ }^{(4)}$.

The management of clubfoot deformity has been transformed in the last two decades from surgical correction (posterior medial release) to the nonsurgical Ponseti method ${ }^{(\mathbf{5})}$.

This study aimed to evaluate the efficacy and safety of percutaneous Achilles tenotomy as an office procedure during the Ponseti technique in the correction of CTEV deformity of the foot.

\section{PATIENTS AND METHODS}

This randomized clinical trial study was conducted in the Orthopedic Outpatient Clinic of Zagazig University Hospitals on 12 patients with idiopathic clubfoot managed with the Ponseti technique during the period from April 2020 to September 2020. We performed the procedure on 12 patients with idiopathic (15 feet) who were included in this prospective study, syndromic cases, recurrent or residual cases were excluded.

Inclusion criteria: Idiopathic clubfoot managed with the Ponseti technique. Age: below 1 year. Exclusion criteria: Syndromic cases such as arthrogryposis multiplex congenita, Spina Bifida, cerebral palsy, and poliomyelitis. Residual talipes equinovarus after Ponseti technique. Recurrent cases after surgical correction.

Written informed consent was obtained from all participants' parents and the study was approved by the research ethics committee of the Faculty of Medicine, Zagazig University. The work has been carried out following The Code of Ethics of the World Medical Association (Declaration of Helsinki) for studies involving humans.

\section{Ethical consent:}

Approval of the study was obtained from Zagazeg University academic and ethical committee. Every 
patient signed informed written consent for the acceptance of the operation.

\section{Pre-operative:}

All patients underwent full history taking, clinical picture, and each patient was subjected to thorough general, physical, and systemic examination including spine, hip, and extremities. After taking a complete history, mobility of the foot was assessed by applying gentle corrective manipulation. The foot was classified into supple type if the manual reduction is possible and rigid type, where manual reduction is impossible.

\section{Surgical technique:}

Babies underwent serial manipulation and casting according to the Ponseti method. The Catterall Pirani score was routinely used for the ascertainment of progression. Tenotomy was indicated while the midfoot is corrected (mid-foot score $=0$ ) and if equinus deformity is still present (ankle dorsiflexion $<10$ degrees). The procedure was performed during routine outpatient clinic activity.

A topical anesthetic cream was applied 30 minutes before the procedure, an oral/rectal analgesia was given, and the baby is fed. A local anesthetic was injected subcutaneously immediately before the procedure. The tendon was completely sectioned by needle thread No (16,18 gauge), the foot must be in a dorsiflexed and abducted position. A long-leg plaster cast was applied immediately, and after 1 hour of supervision, we reviewed the complete course of clubfoot management in each of the babies who underwent Achilles tenotomy.

The baby was placed in the supine position, with the knee flexed to 90 degrees and the hip abducted to make the heel easily accessible. An assistant maintains the position till the procedure is completed. The tendon of Achilles is easily palpated when the foot is forced into dorsiflexion, which makes the tendon tense. With all the aseptic precautions using povidine iodine and $1 \%$ lignocaine of $\sim 0.2 \mathrm{ml}$ given using an insulin syringe, medial border of tendo-Achilles palpated and a 16 gauge sterile needle was inserted from the medial border of the tendon about 1 to $2 \mathrm{~cm}$ proximal to the tendo-Achilles insertion into calcaneum. Sectioning of the tendon performed using the beveled tip of the needle through lateralization and elevation movements of the cutting end (Figure 1).

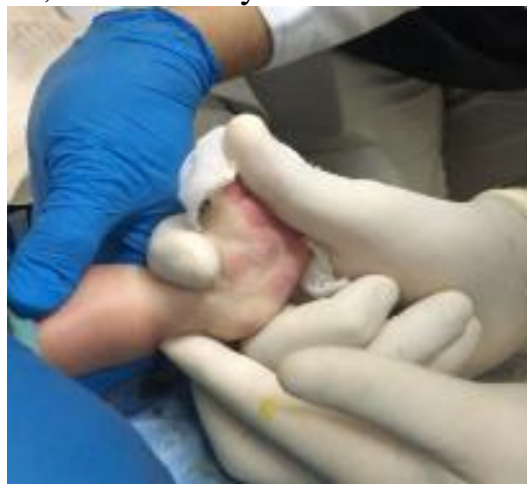

A

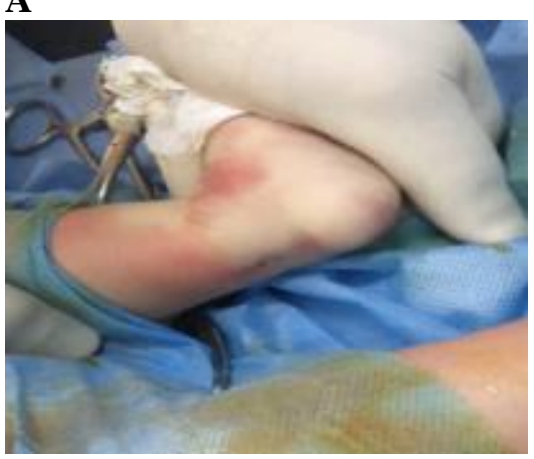

D

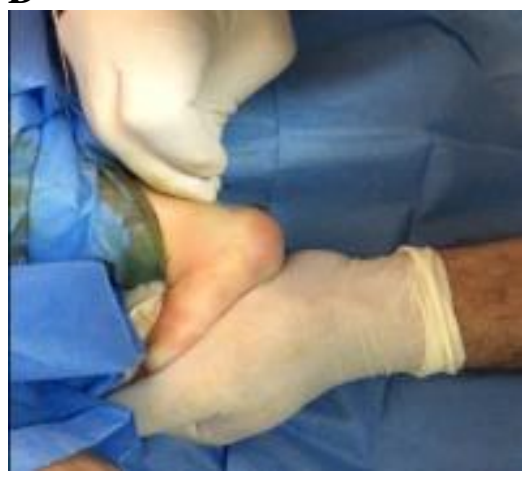

G

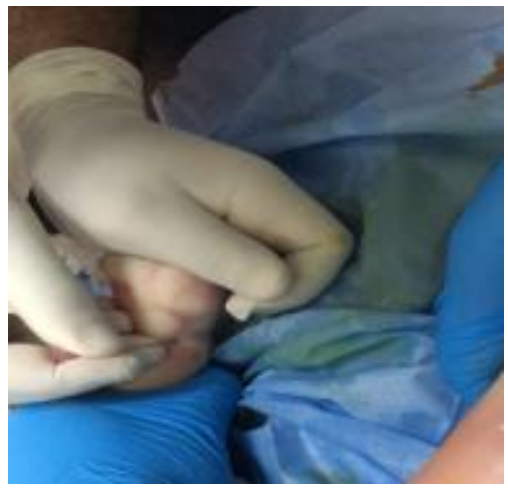

B

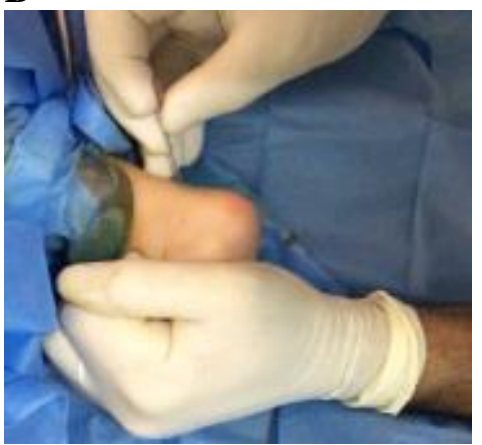

$\mathbf{E}$

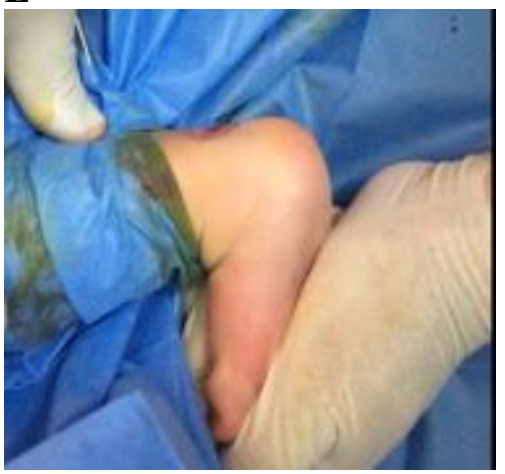

H
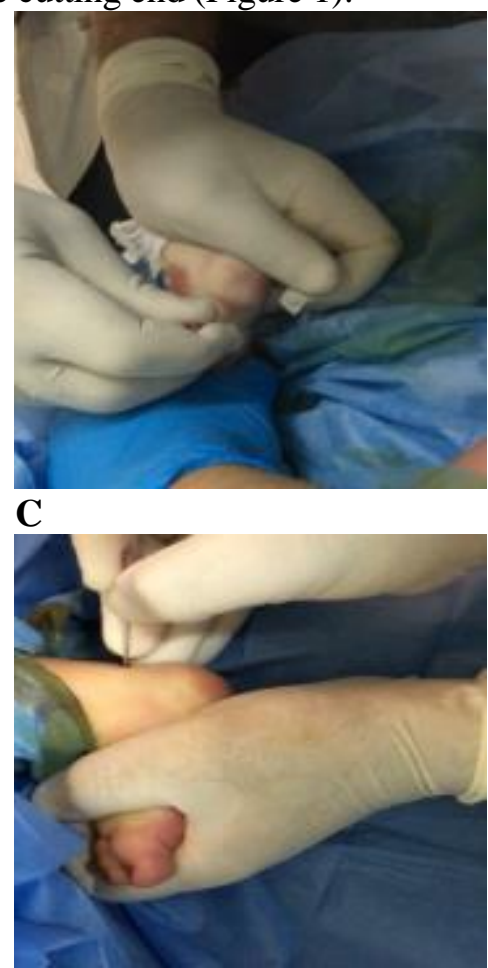

$\mathbf{F}$

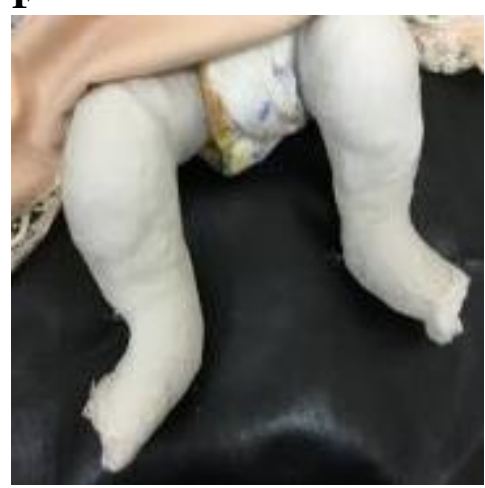

$\mathbf{I}$ 


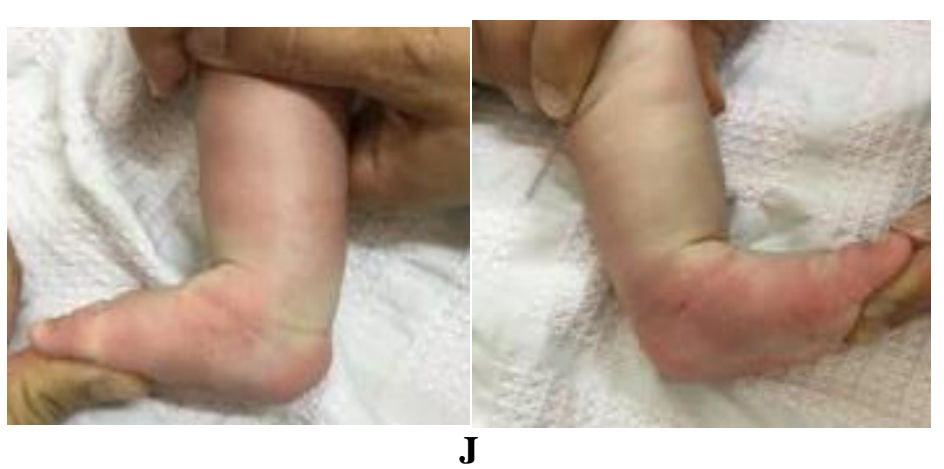

Figure (1): Steps of percutaneous needle tenotomy A: Sterilization of the foot, and palpation of tendo-Achilles of the left foot. B: Introduction of needle medial to the tendon. C: Cutting of tendo-Achilles (tenotomy). D: Improving dorsiflexion after complete tenotomy. E, F, $\mathrm{G}, \mathrm{H}$ : The same tenotomy steps for the right foot. I: Casting after by bilateral tenotomy. J: After cast removal 3 weeks after tenotomy.

\section{Post-operative follow up:}

Removal of the post-tenotomy cast. Fitted into foot abduction brace (to be worn for 23 hours per day). The period of follow-up was for six months. Assessment of residual equinus or repeated tenotomy was specifically sought.

\section{Statistical analysis}

Data collected throughout history, basic clinical examination, laboratory investigations, and outcome measures coded, entered, and analyzed using Microsoft Excel software. Data were then imported into Statistical Package for the Social Sciences (SPSS version 20.0) (Statistical Package for the Social Sciences) software for analysis. P-value was set at $<0.05$ for significant results and $<0.001$ for a highly significant result.

\section{RESULTS}

The mean age at the time of presentation was 10.83 \pm 2.08 days with a minimum of 7 and a maximum of 14 Days. As regards sex distribution males represent $58.3 \%$ and females $41.7 \%$. As regards the side distribution; six cases $(50 \%)$ were right, three $(25 \%)$ were left and three $(25 \%)$ were bilateral (Table 1).

Table (1): Age and sex distribution among the studied group.

\begin{tabular}{|c|c|c|c|}
\hline \multirow{3}{*}{ Age / days } & \multirow{3}{*}{$\begin{array}{c}\text { Mean } \pm \text { SD } \\
\text { Median (Range) }\end{array}$} & \multirow{2}{*}{\multicolumn{2}{|c|}{$\frac{10.83 \pm 2.08}{12.0(7-14)}$}} \\
\hline & & & \\
\hline & & $\mathbf{N}$ & $\%$ \\
\hline \multirow{2}{*}{ Sex } & Male & 7 & 58.3 \\
\hline & Female & 5 & $\begin{array}{l}41.7 \\
\end{array}$ \\
\hline \multicolumn{2}{|r|}{ Total } & 12 & 100.0 \\
\hline \multicolumn{2}{|c|}{$\begin{array}{c}\text { Side distribution among the } \\
\text { studied group }\end{array}$} & $\mathbf{N}$ & $\%$ \\
\hline & Left & 3 & 25.0 \\
\hline \multirow[t]{2}{*}{ Side } & Right & 6 & 50.0 \\
\hline & Bilateral & 3 & 25.0 \\
\hline \multicolumn{2}{|r|}{ Total } & 12 & 100.0 \\
\hline
\end{tabular}

There was a statistically significant decrease of the mid and hind foot contracture score from pre to 3 months of follow up and also a significant decrease between pre and 6 months of follow up score but no significant difference was found between scores of 3 months and 6 months (Table 2).

Table (2): MID, Hind foot contracture score distribution at pre, 3 and 6 months of follow up.

\begin{tabular}{|c|c|c|c|c|}
\hline$\overline{\mathrm{MID}}$ & Mean & $\overline{S D}$ & Paired $\mathbf{t}$ & P-value \\
\hline MFCS_PRE & 2.5833 & 0.51493 & \multirow{2}{*}{11.406} & \multirow{2}{*}{$0.00 * *$} \\
\hline MFCS_3months & 0.6417 & 0.21569 & & \\
\hline MFCS_PRE & 2.5833 & 0.51493 & \multirow{2}{*}{10.352} & \multirow{2}{*}{$0.00 * *$} \\
\hline MFCS_6months & 0.4167 & 0.13340 & & \\
\hline MFCS_3months & 0.6417 & 0.21569 & \multirow{2}{*}{0.897} & \multirow{2}{*}{0.389} \\
\hline MFCS_6months & 0.4167 & 0.13340 & & \\
\hline Hind foot & Mean & SD & Paired $\mathbf{t}$ & P-value \\
\hline HFCS_PRE & 2.5417 & 0.39648 & \multirow{2}{*}{17.861} & \multirow{2}{*}{$0.00 * *$} \\
\hline HFCS_3months & 0.5417 & 0.19810 & & \\
\hline HFCS_PRE & 2.5417 & 0.39648 & \multirow{2}{*}{9.398} & \multirow{2}{*}{$0.00 * *$} \\
\hline HFCS_6months & 0.5021 & 0.12621 & & \\
\hline HFCS_3months & 0.5417 & 0.19810 & \multirow{2}{*}{0.220} & \multirow{2}{*}{0.830} \\
\hline HFCS_6months & 0.5021 & 0.12621 & & \\
\hline
\end{tabular}

In this study, there was a statistically significant decrease of total Piriani score from pre to 3 months of follow up and also a significant decrease between pre and 6 months of follow up score but no significant difference was found between scores of 3 and 6 months (Table 3 ). 
Table (3): Total Piriani score distribution at pre, 3 months, and 6 months of follow-up.

\begin{tabular}{|c|c|c|c|c|}
\hline & Mean & $\begin{array}{c}\text { Std. } \\
\text { Deviation }\end{array}$ & \multirow{2}{*}{ Paired t } & P-value \\
\hline \hline Total Piriani score pre & 5.1250 & 0.85613 & \multirow{2}{*}{12.310} & \multirow{2}{*}{$0.001 * *$} \\
\hline Total Piriani score 3months & 1.0833 & 0.37312 & & \multirow{2}{*}{$0.001 * *$} \\
\hline Total_Piriani_score_pre & 5.1250 & 0.85613 & \multirow{2}{*}{0.372} & \multirow{2}{*}{0.474} \\
\hline Total_Piriani_score_6months & 0.9167 & 0.32007 & 0.742 & \\
\hline Total_piriani_score_3months & 1.0833 & 0.37312 & & \\
\hline Total_piriani_score_6months & 0.9167 & 0.32007 & & \\
\hline
\end{tabular}

In this study, there was a statistically significant increase of talocalcaneal angle (TCA) from pre to 3 months of follow up and also a significant increase between pre and 6 months of follow up score but no significant difference was found between scores of 3 and 6 months (Table 4).

Table (4): Radiological assessment (TCA) distribution at pre, 3, and 6 months of follow-up.

\begin{tabular}{|c|c|c|c|c|}
\hline & Mean & Std. Deviation & Paired t & $\mathbf{P}$ \\
\hline TCA pre & 20.8000 & 1.52128 & \multirow{2}{*}{20.805} & \multirow{2}{*}{$0.001 * *$} \\
\hline TCA post 3 months & 30.4667 & 1.72654 & & \\
\hline TCA pre & 20.8000 & 1.52128 & \multirow{2}{*}{19.552} & \multirow{2}{*}{$0.001 * *$} \\
\hline TCA post 6 months & 29.3521 & 2.03072 & & \\
\hline TCA post 3 months & 30.4667 & 1.72654 & \multirow{2}{*}{0.856} & \multirow{2}{*}{0.423} \\
\hline TCA post 6 months & 30.3521 & 2.03072 & & \\
\hline
\end{tabular}

In this study, there was a statistically significant decrease from pre to 3 months of follow up and also a significant decrease between pre and 6 months of follow up score but no significant difference was found between scores of 3 months and 6 months (Table 5)

Table (5): Radiological assessment TIC- lateral tibial calcaneal angle distribution at pre, 3, and 6 months of follow up.

\begin{tabular}{|c|c|c|c|c|}
\hline & Mean & Std. Deviation & Paired t & P \\
\hline \hline TiC pre & 102.8000 & 5.01711 & \multirow{2}{*}{26.531} & \multirow{2}{*}{$0.001 * *$} \\
\hline TiC post 3 months & 62.4667 & 4.50185 & & \multirow{2}{*}{$0.001 * *$} \\
\hline TiC pre & 102.8000 & 5.01711 & \multirow{2}{*}{19.769} & \multirow{2}{*}{0.288} \\
\hline TiC post 6 months & 63.4667 & 7.52962 & \multirow{2}{*}{1.105} & \\
\hline TiC post 3 months & 62.4667 & 4.50185 & 7.52962 & \\
\hline TiC post 6 months & 63.4667 & & & \\
\hline
\end{tabular}

As regards the complications in this study, there were two feet of relapse (13.3\%), one foot relapsed equinus deformity, and one adducts foot deformity (Table 6).

Table (6): Complication distribution among studied group (15 feet).

\begin{tabular}{|c|c|c|c|}
\hline \multicolumn{2}{|c|}{} & $\mathbf{N}$ & \% \\
\hline \hline \multirow{3}{*}{ Relapse } & No & 13 & 86.7 \\
\cline { 2 - 4 } & Yes & 2 & 13.3 \\
\cline { 2 - 4 } & Total & 15 & 100.0 \\
\hline
\end{tabular}

As regards the outcome, 13 feet had a favorable outcome (8 cases excellent and 5 cases good) and 2 feet had a fair outcome (Table 7).

Table (7): Outcome (according to Piriani) distribution among the studied group.

\begin{tabular}{|c|c|c|c|}
\hline \multicolumn{2}{|c|}{ Piriani score } & N & \% \\
\hline \hline \multirow{3}{*}{ Outcome } & Fair & 2 & 13.4 \\
\cline { 2 - 4 } & Good & 5 & 33.3 \\
\cline { 2 - 4 } & Excellent & 8 & 53.3 \\
\cline { 2 - 4 } & Total & 15 & 100.0 \\
\hline
\end{tabular}




\section{DISCUSSION}

To better management of club foot, we performed this prospective randomized clinical study in the Orthopedic Outpatient Clinic of Zagazig University Hospitals which included 15 feet in 12 children with an average age at presentation of $10.83 \pm 2.08$ with a minimum of 7 , and a maximum of 14 days. Regarding sex distribution males represent $58.3 \%$ and females were $41.7 \%$ with a male to female ratio of 7:5.50\% of cases were right-sided, $25 \%$ were left and $25.0 \%$ were bilateral. This was in agreement with a study conducted by Agius et al. ${ }^{(6)}$ that included 59 clubfeet (40 babies, 19 bilateral cases) with a diagnosis of idiopathic congenital clubfoot. Tenotomy was performed at an average post-natal age of 10.5 weeks in the clinic group and 12.1 weeks in the theatre group The ratio of males to females was 2:1.

Additionally, Porecha et al. ${ }^{(7)}$ reported in their study that a total number of 49 patients with 67 clubfeet were treated and followed for a mean of five years. 39 of them $(79.59 \%)$ were males, thus male to female ratio was $3: 9$. Only 18 patients $(36.73 \%)$ had bilateral involvement while 31 patients $(63.27 \%)$ had unilateral involvement out of which 17 (54.84\%) had right foot, and 14 (45.16\%) had left foot involvement. No relationship was found with birth order or family history. At the beginning of the treatment, 42 patients $(85.71 \%)$ are between $0-12$ weeks of age (mean 2 weeks), 5 patients $(10.20 \%)$ are in between 13-24 weeks of age (mean 15 weeks) while $2(4.08 \%)$ patient are in between 25-36 weeks of age (mean 34 weeks).

There was a significant decrease in mid and hind foot contracture score and total Piriani from pre to 3 months of follow up and also a significant decrease between pre and 6 months of follow up score but no significant difference was found between scores of 3 , and 6 months $(2.58 \pm 0.51,0.64 \pm 0.21$ and $0.41 \pm 0.13$ ), $(2.54 \pm 0.39,0.54 \pm 0.19$ and $0.50 \pm 0.12)$ and $(5.12 \pm 0.8$, $1.08 \pm 0.37$ and $0.91 \pm 0.32$ ) respectively.

Porecha et al. (7) found that at the commencement of treatment, of the 18 bilateral clubfeet patients ( 36 clubfeet) 17 children ( 34 clubfeet) had a Pirani severity score of six, and one child (2 clubfeet) had a Pirani score of five. In the unilateral group, the mean Pirani score was 5.83 (range 5-6). The mean mid-foot score and hindfoot score for the entire group was 2.8 (range 2.5-3) and 2.76 (range 2-3) respectively. The mean number of the casts that were applied to obtain correction was 6.8 (range 6-8). The more severe the initial deformity and the treatment initiation after 12 weeks of the age, the more casts were required to obtain correction. 47 children $(95.91 \%)$ needed percutaneous tenotomy, 18 in the bilateral group and 29 in the unilateral group. The mean midfoot score and hindfoot score for the entire group at the time of equinus deformity was 0.5 and 2.5 respectively.
There was no delay between final cast removal and fitting of the D-B splint.

Regarding radiological assessment talocalcaneal angle (TCA), there was a significant increase from pre to 3 months of follow up and also a significant increase between pre and 6 months of follow up score but no significant difference was found between scores of 3 , and 6 months $(20.8 \pm 1.5$, $30.47 \pm 1.7$ and $29.3 \pm 2.03$ ) while regarding lateral tibio calcaneal angle (TIC), there was a statistically significant decrease from pre to 3 months of follow up and also significant decrease between pre and 6 months of follow up score but no significant difference was found between scores of 3 , and 6 months $(102.8 \pm 5.02,62.47 \pm 4.5$ and $63.46 \pm 7.5)$ respectively ${ }^{(6)}$.

Concerning the outcome, after completion of treatment in the present study, 13 feet had a favorable outcome ( 8 cases $(53.3 \%)$ excellent and 5 cases good $33.3 \%$ ) and 2 feet had fair outcome (13.3). These 2 feet were relapsed (13.3\%), one foot relapsed equinus deformity, and one adducts foot deformity, The equinus deformity patient responded to repeated tendo-Achilles tenotomy and further Ponseti casting and adductus foot deformity responded to adductor tenotomy and cast. Both two cases obtained satisfactory outcomes after the completion of treatment. There was no significant association between the outcome and the children's characteristics.

These findings were similar to that of Porecha et al. ${ }^{(7)}$ where the mean duration of the treatment up to the application of the D-B Splint was 9.6 weeks. The initial correction was obtained in all 67 clubfeet $(100 \%)$ with the Ponseti method. Fourteen children $28.57 \%$ (19 feet 28.35\%) had a relapse of the deformity.

Opposite to our findings, Gerlach et al. ${ }^{(8)}$ observed relapse in $68 \%$ (19 of 28 feet) after a mean of 7.1 months mostly treated with reapplication of the Ponseti method.

Oppositely Agius et al. ${ }^{(\boldsymbol{6})}$ found in their study that a satisfactory rate was among 6 cases from 19 $(32 \%)$ by the parental satisfaction survey.

Another study prospectively evaluated 55 clubfeet (37 patients) to determine clinically whether the Ponseti method was effective in the management of clubfoot in older children between the age of 12 and 36 months (mean: 24.8 months). All the patients belonged to moderate or severe grades of deformity as per the Pirani scoring. Painless, supple, plantigrade, and cosmetically acceptable feet were achieved in 49 clubfeet. Seven patients (seven feet) developed recurrence of adduction, varus, and equinus deformity whereas three patients (five feet) developed isolated recurrence of equinus deformity. These seven patients responded to repeat treatment and obtained satisfactory outcomes. Four of these seven patients 
underwent tibialis anterior transfer to the third cuneiform for dynamic supination. Three patients, who developed isolated recurrence of equinus deformity, underwent repeat tenotomy. One foot achieved a satisfactory amount of dorsiflexion, three feet underwent tendo-Achilles lengthening whereas another foot underwent posterior release to obtain satisfactory dorsiflexion. Six to 12 casts (mean=10) were required to obtain correction of clubfoot deformities. The mean period of immobilization in a cast was 13.9 weeks (10-15 weeks). They reported that the Ponseti method is effective in children between the age of 12 and 36 months $^{(9)}$.

In a retrospective, consecutive review over 10 years in a tertiary center. A total of 11 children with 18 myelomeningocele-associated clubfeet were included, with an average follow-up duration of 4.5 years (range 3-9 years). The average age at presentation was 4.7 weeks, with an average Pirani score of 5.5. An initial correction was achieved in all children with an average of 7 (range 4-9) Ponseti casts and tendo-Achilles tenotomy was performed in 17 of 18 feet $(94.4 \%)$. Nine children with 15 of $18 \quad(83.3 \%)$ myelomeningocele-associated clubfeet achieved a satisfactory outcome at the final follow-up, with functional, pain-free feet. Recurrence occurred in five of $15(33.3 \%)$ feet which was managed successfully with a second tendo-Achilles tenotomy and further Ponseti casting. Two children three of 18 (16.7\%) failed Ponseti treatment ${ }^{(9)}$.

\section{CONCLUSION}

The Ponseti method is an effective first-line treatment for syndrome-associated talipes equinovarus to achieve functional painless feet.

Ponseti method is safe and effective for the correction of complex clubfeet. Early diagnosis and strict adherence to the Ponseti principles are keys to achieve deformity correction. Patients with complex clubfoot require frequent follow-up because of a higher recurrence rate which can be repaired by repetition of the procedure.

\section{REFERENCES}

1. Famude S, Adewole O, Komolafe O (2016): Achievement of developmental milestones in children with idiopathic clubfoot treated with Ponseti method in Lagos Nigeria. African Journal of Physiotherapy and Rehabilitation Sciences, 8(1-2): 44-46.

2. Chang C, Wang S, Kuo K (2019): The Ponseti Method Decreased the Surgical Incidence in Children with Congenital Clubfoot: A Population-Based, 8 Birth-Year Cohort Study. JBJS., 101(21):1955-1960.

3. Basit $\mathbf{S}$, Khoshhal K (2018): Genetics of clubfoot; recent progress and future perspectives. European Journal of Medical Genetics, 61(2):107-113.

4. Turco V (1979): Resistant congenital clubfoot: onestage posteromedial release with internal fixation. J Bone Joint Surg., 61:805-812.

5. Sattar A, Shabbir M, Rehman I et al. (2019): Efficacy of percutaneous needle tenotomy during ponseti technique in the management of congenital club foot deformity in children. Pak J Surg., 35(4):316-319.

6. Agius L, Wickham A, Knudsen $J$ et al. (2018): Achilles tenotomy as an office procedure and current practicing trends among New Zealand orthopedic surgeons. The New Zealand Medical Journal (Online), 131(1475): 44-50.

7. Porecha M, Parmar S, Chavda R (2011): Mid-term results of Ponseti method for the treatment of congenital idiopathic clubfoot-(a study of 67 clubfeet with mean five-year follow-up). Journal of Orthopaedic Surgery and Research , 6(1):1-7.

8. Gerlach D, Gurnett C, Limpaphayom N et al. (2019): Early results of the Ponseti method for the treatment of club foot associated with myelomeningocele. J Bone Joint Surg Am., 91(6):1350-1359.

9. Ponseti I (1996): Congenital Clubfoot: Fundamentals of Treatment. New York: Oxford University Press; Pp. $37-48$. 